\title{
Characterization of the Japanese Manage- ment Technique 5S. Polytechnic Dining Room Case
}

\section{Caracterización de la Técnica de Gestión Japonesa 5S. Caso Comedor Politécnico}

VII International Congress of Science, Technology, Entrepreneurship and Innovation (SECTEI 2020)

Corresponding Author:

M. Izurieta

oparadag@ulvr.edu.ec

Published: 26 August 2021

Production and Hosting by Knowledge E

(c) M. Izurieta et al. This article is distributed under the terms of the Creative Commons Attribution License, which permits unrestricted use and redistribution provided that the original author and source are credited.

\section{Izurieta ${ }^{1}$, G. Miño ${ }^{2}$, and D. Vallejo ${ }^{1}$}

${ }^{1}$ Facultad de Administración de Empresas, Escuela Superior Politécnica de Chimborazo, Riobamba, Ecuador

${ }^{2}$ Vicerrectora Académica, Escuela Superior Politécnica de Chimborazo, Riobamba, Ecuador

\section{Abstract}

This research aimed to characterize the Japanese $5 S$ management technique, using a case study of the Polytechnic Dining Room, given that in this unit you can apply the 5S technique without any problem, and improve quality, productivity and competitiveness. Several elements were examined including the existence of work material, procedures for eliminating unnecessary materials, location of work materials, cleaning of work areas, standardization of processes, use of uniforms, and protective equipment, among others. Qualitative and quantitative approaches were used. A documentary research instrument was applied, using a Likert scale with ordinal variables. According to the results, those that obtained the highest rating of 7 were as follows: Seiris (classification) 25\%, Seiton (Order) 36\%, Seiso (Cleaning) 32\%, Seiketsu (Standardization) 36\%, and Shitsuke (Maintenance) 57\%. Therefore, we conclude that in Shitsuke there was greater employee commitment, and Seiri obtained the lowest qualification.

Keywords: 5S, quality improvement, productivity, competitiveness.

\section{Resumen}

Con la finalidad de conocer los elementos innecesarios, existencia de material de trabajo, procedimientos de eliminación de materiales innecesarios, ubicación de materiales de trabajo, limpieza de áreas de trabajo, estandarización de procesos, utilización de uniformes, equipo de protección entre otros, la presente investigación tienen el objetivo de caracterizar la técnica de gestión japonesa 5S, caso Comedor Politécnico, considerando que en esta dependencia puede aplicar sin ninguna problema la técnica 5S, mejorar la calidad, su productividad y competitividad; la investigación tiene un enfoque cualicuantitativo, con un método analítico sintético con un nivel de investigación exploratorio, descriptivo, se aplica un instrumento de investigación documental, de escala con variables ordinales, utilizando la escala Likert, método inductivo empírico de información primaria, en la cual se obtiene la siguientes conclusiones obteniendo la calificación más alta de 7, en lo referente Seiri (clasificación) 25\%, Seiton (Orden)36\%, Seiso (Limpieza) 32\%, Seiketsu (Estandarización) 36\%, Shitsuke (Mantenimiento) $57 \%$, de esta manera de concluye que en Shitsuke donde existe mayor compromiso de los empleados, y en Seiri es la que menor calificación se obtiene.

Palabras Clave: 5S, mejora de la calidad, productividad, competitividad. 


\section{Introducción}

En todas las organizaciones al hablar de mejoramiento continuo, se pretende implementar soluciones muchas veces complicadas al considerar conceptos que están ligados íntimamente con la gestión como son organizar, ordenar y limpiar, estas actividades que son realizadas empíricamente muchas veces son de hábito doméstico y nunca empresarial; estas actividades son fundamentales para poder identificar problemas y procesos, si no se cuenta con un lugar bien limpio y bien ordenado; si no se estandarizan procesos y no existe disciplina la organización se convierte en una zona de peligro [1].

Las $5 \mathrm{~S}$ es una filosofía de trabajo que permite desarrollar un comportamiento sistemático para mantener continuamente la clasificación, el orden y la limpieza, lo que permite de forma inmediata una mayor productividad, mejorar la seguridad, el clima laboral, la motivación del personal, la calidad, la eficiencia y, en consecuencia, la competitividad de la organización [2].

La efectividad del trabajo en una organización se la obtiene al mantener un área limpia, reduciendo el riesgo potencial de que se produzca accidentes; mejorando el bienestar físico y mental del trabajador, incrementando la vida útil del equipo al evitar su deterioro por contaminación y suciedad [3].

La filosofía 5S (housekeeping) toma su nombre de cinco palabras japonesas: Seiri, seiton, seiso, seiketsu y shitsuke [4] en la cual se establece los siguientes puntos:

La 1era Seiri, implica seleccionar; separando elementos necesarios de los innecesarios

Este principio consiste en retirar o desechar los materiales o elemento que no ayuda a realizar una labor, este principio se puede aplicar en todas las áreas ya sea en áreas administrativas o de producción.

La '2nda Seiton', 'permite ordenar/organizar los elementos necesarios en el lugar de trabajo'.

Este principio se basa más en la apariencia y la parte físico del entorno donde se está operando, lo cual, este principio nos da a conocer la importancia de colocar cada elemento en un lugar correspondiente para tener mejor acceso a cada uno de los elementos. Un lugar para cada cosa y cada cosa en su lugar.

La '3era Seiso', 'significa limpiar y asear el entorno para anticiparse a los problemas'.

La importancia de limpieza no está en limpiar o dar manteniendo a los equipos de trabajo, sino que también ayuda a detectar fallas que no se hallan visto y así mismo mejorar y estar más seguros del ambiente y lugar de trabajo ya que evita malos olores.

La '4ta Seiketsu', 'permite estandarizar las normas generales por los equipos'.

Este principio busca la estandarización de la limpieza y orden y solo se puede llevar a cabo si se cumplen las primeras S. para llevar a cabo esta estandarización un técnico 
realiza el análisis y la observación del lugar, lo más favorable es tomar una fotografía del área para que los trabajadores dejen el lugar como lo reciben.

La 5ta Shitsuke, 'dinamiza las auditorias de seguimiento y consolida el ámbito de la mejora'.

Este principio busca que todo el procedimiento o las 4S anteriormente mencionado no se rompan o perduren en el tiempo y si es posible mejorar continuamente, por lo cual es importante que se lleve un control periódico, realizar visitas sorpresas a las distintas aéreas de operación ya que ayudará a estandarizar las 4S anteriores.

Practicar las $5 \mathrm{~S}$ se ha vuelto casi indispensable para cualquier empresa que participa en manufactura o servicios. Las actividades comprenden. (A) Separa todo lo innecesario y eliminarlo. (B) Poner en orden los elementos esenciales, de manera que se tenga fácil acceso en éstos. (C) limpiar todo - herramientas y lugares de trabajo - removiendo manchas, mugre, desperdicios y erradicando fuentes de suciedad. (D) Llevar a cabo una rutina de limpieza y verificación y (E) Estandarización los cuatro pasos anteriores para constituir un proceso sin fin y que pueda mejorarse [5].

Un ambiente ordenado en el comedor aumentaría la productividad a través de la mejora de los procesos y reducción de los riesgos laborales, involucrando a todos los empleados de manera que tengan objetivos enfocados a un propósito en común, ayudaría en la reducción de desperdicios, mejor manejo u organización para el uso de los espacios, aumentaría la comunicación interna, eliminaría elementos que no son necesarios dentro de los procesos administrativos y de producción y además de ser ordenado reduce el tiempo de búsqueda de loe elementos.

Al momento de emplear el principio de la limpieza se garantiza el aseo del área de trabajo y se genera mayor control sobre los defectos que pueden existir

El beneficio de la estandarización es el poder establecer los requisitos y la disciplina necesaria para garantiza la reducción de errores y fortalecer la interacción entre colaboradores. Por lo tanto el uso de estas buenas prácticas como efecto resulta un mejor servicio del comedor para los estudiantes, dando como resultado la satisfacción de los estudiantes politécnicos beneficiarios del servicio de alimentación.

Principios del Modelo de Mejora Continua: La mejora continua como proceso de resolución de problemas mediante la cual la organización alcanza un nuevo nivel de desempeño, que a su vez es el punto de partida para un nuevo esfuerzo de mejoramiento, toma como centro el cliente y se orienta a los cambios graduales, pero creando una cultura de perfeccionamiento en la organización orientada a las personas mas que a la tecnología por lo que se requiere poca inversión pero un gran esfuerzo mara mantenerlos [6].

El objetivo del modelo de gestión a través de la mejora de la calidad es disponer de un enfoque sistemático, motivado por las necesidades y percepciones de los clientes, que dirija a todos los elementos de la organización a alcanzar niveles de desempeño 
superiores en procesos, productos y servicios. Los objetivos específicos del modelo son:

1. Lograr de manera sistemática el mejoramiento del desempeño de la organización, gestionando el cambio.

2. Lograr que los objetivos de la organización se correspondan con los objetivos de las áreas, fijando metas de mejoras claras y objetivos medibles.

3. Identificar los procesos claves de la organización para lograr el éxito.

4. Servir de retroalimentación para evaluar la vigencia de las estrategias formuladas.

5. Identificar y seleccionar las áreas de mejoras prioritarias.

Mejoramiento Continuo de los procesos según Álvarez [7]:

Secuencia de actividades orientadas a generar un valor añadido sobre una entrada para conseguir un resultado, y una salida que a su vez satisfaga los requerimientos del cliente.

Secuencias ordenadas y lógicas de actividades de transformación, que parten de unas entradas (informaciones en una sentido amplio (pedidos datos, especificaciones), más medios materiales (máquinas, equipos, materias primas, consumibles) para alcanzar los resultados programados, que se entregan a quienes los han solicitado, los clientes de cada proceso.

Secuencia ordenada de actividades repetitivas que se realizan en la organización por una persona, grupo o de departamento, con la capacidad de transformar unas entradas (inputs) en salidas o resultados programados (outputs) para su destinatario (dentro o fuera de la empresa que ha solicitado y que son los clientes de cada proceso) con un valor agregado [7].

Por otra parte, la palabra Productividad apareció por primera vez en un artículo de Quesnay en el año 1766, en la tabla I se recogen diferentes definiciones de este término [8].

Así mismo, se tienen las siguientes definiciones de autores de las dos últimas décadas:

1. 'Es el resultado final de un complejo proceso social, compuesto por ciencia, investigación y desarrollo, educación, tecnología, dirección de empresa, medios de producción y organización de trabajadores' [9].

2. 'Es la relación entre la producción obtenida por un sistema de producción o servicios y los recursos utilizados para obtenerla' [10].

3. 'La productividad es el producto final del esfuerzo y combinación de todos los recursos humanos, materiales y financieros que integran una empresa' [11]. 


\section{Table 1}

Definiciones de productividad.

\begin{tabular}{|c|c|c|}
\hline Autor & Año & Definición \\
\hline Quesnay & 1766 & La palabra productividad aparece por primera vez. \\
\hline Litré & 1883 & Facultad de producir \\
\hline Early & 1900 & $\begin{array}{l}\text { Relación entre producción y los medios empleados } \\
\text { para lograrla }\end{array}$ \\
\hline OEEC & 1950 & $\begin{array}{l}\text { Cociente que se obtiene al dividir la producción por } \\
\text { uno de los factores de producción }\end{array}$ \\
\hline Davis & 1955 & Cambio en el producto por los recursos gastados \\
\hline Fabricant & 1962 & $\begin{array}{l}\text { Siempre una razón entre la producción y los } \\
\text { insumos }\end{array}$ \\
\hline Kendrick y Creamer & 1965 & $\begin{array}{l}\text { Definiciones funcionales para la productividad } \\
\text { parcial, de factor total y total }\end{array}$ \\
\hline Siegel & 1976 & Una familia de razones \\
\hline Sumanth & 1979 & $\begin{array}{l}\text { Productividad total. La razón de producción tangible } \\
\text { entre insumos tangibles. }\end{array}$ \\
\hline
\end{tabular}

Elaborado por: Autores.

Fuente: Definiciones de Productividad [8].

4. La productividad tiene que ver con los resultados que se obtienen en un proceso, por lo que incrementar la productividad es lograr mejores resultados considerando los recursos empleados para generarlos [12].

5. La productividad es una expresión de cuán eficientemente los bienes y servicios están siendo producidos. Es por eso que la productividad se expresa en unidades físicas o económicas, en las cantidades o los valores (dinero) [13].

La revisión de las distintas definiciones, permiten asumir la Productividad en esta investigación, como la integración de las variables gente, tecnología y dinero con el fin de generar bienes y servicios, que sean beneficiosos para todos los actores involucrados (empresa, trabajadores, clientes y sociedad), resaltando así la importancia de lo social, para lo cual es necesario que las personas tengan conciencia de la importancia de la productividad para mejorar la calidad de vida y la organización por su parte, valorar el rol insustituible de la gente para lograr incrementos en la productividad [13].

Cuando se enfatiza en que la productividad es 'hacer más con menos' ó una 'relación entre las salidas y las entradas', generalmente se asume el término como sinónimo de eficiencia, eficacia o efectividad organizacional, por lo que es necesario definir estos términos, que son los más utilizados para evaluar el desempeño de un sistema y que están relacionados con la productividad, pero que no son sinónimos.

Para complementar la presente investigación es necesario hablar de Competitividad como es una reconsideración de un gran conjunto de indicadores, ninguno de los 
cuales cuenta la historia total, sino que juntos proveen un foco de atención altamente legítimo.

Significa que la competitividad es fundamental para suministrar las condiciones necesarias para el crecimiento y desarrollo empresarial e institucional esto se hace extensivo a las regiones y países.

La competitividad consiste en sostener y expandir la participación de los agentes económicos en distintos sectores con el objetivo de mejorar el nivel de vida de la población. Pero debe tomarse en cuenta que la competitividad debe ir acompañada de la creación de ventajas competitivas aprovechando las condiciones y medios de las regiones del país.

Para la Comisión Económica para América Latina CEPAL, los principales factores a considerar al momento de realizar un análisis de competitividad se detallan [15].

\section{Table 2}

Principales factores de competitividad CEPAL.

Economía
Gobierno e Instituciones
Finanzas
Infraestructura de Información y Comunicación
Recurso Humano
Ciencia y Tecnología
Internacionalización de la Economía
Medio Ambiente

Elaborado por: Autores.

Fuente: Principales factores de competitividad CEPAL [15]

\section{Materiales y Métodos}

Este trabajo investigativo contó con una investigación de campo con un enfoque cualicuantitavo, con métodos analítico-sintético, con la técnica aplicada de Linkert, con 7 items, donde 1 es el valor más bajo y 7 el valor más alto. La misma que ayudó con la recolección de datos, ya que permitió la graduación de la opinión de las personas encuestadas, que midió una actitud que utilizó una escala compuesta por distintas afirmaciones o ítems que permitieron obtener la opinión por parte del personal del comedor politécnico en estudio, se representan en gráficos de dispersión donde en el eje $\mathrm{X}$ se consideran los ítems de Likert, el eje $\mathrm{Y}$ la frecuencia relativa obtenida.

Luego de obtener los datos, luego de aplicar la encuesta con el método líkert se obtienen los siguientes resultados, mismos que se representan en gráficos de 
Table 3

Datos obtenidos encuesta 5S. Caso Comedor Politécnico.

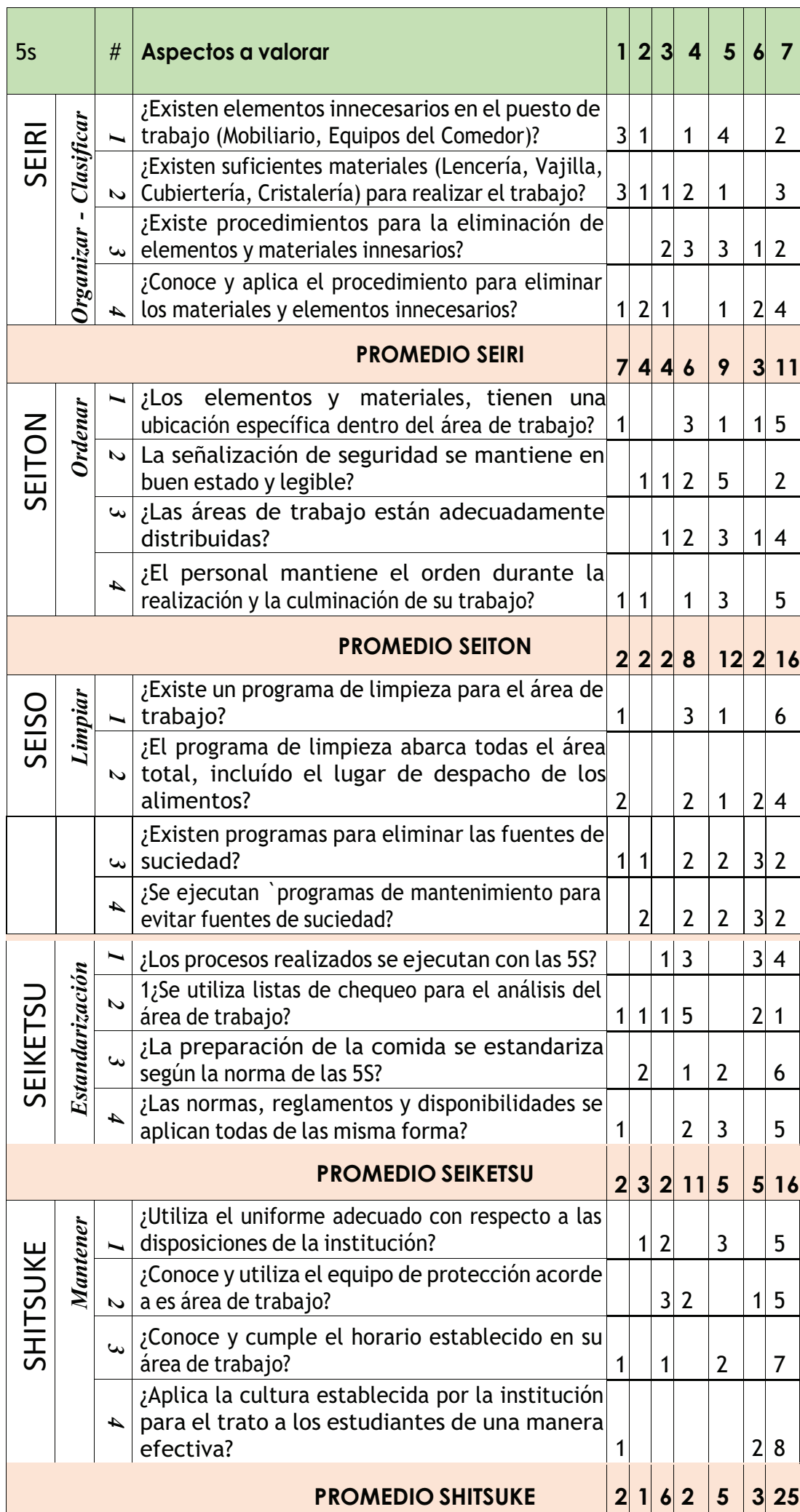

Elaborado por: Autores.

Fuente: Datos obtenidos encuesta 5S, Caso Comedor Politécnico. 
dispersión donde en el eje de la X se consideran los ítems de la encuesta aplicada del 1 al 7 y en el eje $Y$, el número de encuestas realizadas.

SEIRI: Con respecto a clasificación de los materiales utilizados en el comedor politécnico se obtienen resultados no tan homogéneos como se esperaba, el $16 \%$ de los encuestados otorga una calificación de 1, el 9\% una calificación de 2; el 9\% una calificación de 3; el 14\% una calificación de 4; el

$20 \%$ una calificación de 5; el 7\% una calificación de 6 y el 25\% de los encuestados una calificación de 7. En preguntas relacionadas a material innecesario en el puesto de trabajo, suficiencia de materiales para realizar el trabajo, procedimientos para la eliminación elementos y materiales incensarios.

\section{Table 4}

Datos obtenidos encuesta 5S SEIRI, Caso Comedor Politécnico.

\begin{tabular}{l|l|l|l|l|l|l|l}
\hline & 1 & 2 & 3 & 4 & 5 & 6 & 7 \\
Frecuencias absolutas & 7 & 4 & 4 & 6 & 9 & 3 & 11 \\
\hline Frecuencas relativas & $16 \%$ & $9 \%$ & $9 \%$ & $14 \%$ & $20 \%$ & $7 \%$ & $25 \%$
\end{tabular}

Elaborado por: Autores.

Fuente: Datos obtenidos encuesta 5S SEIRI, Caso Comedor Politécnico.

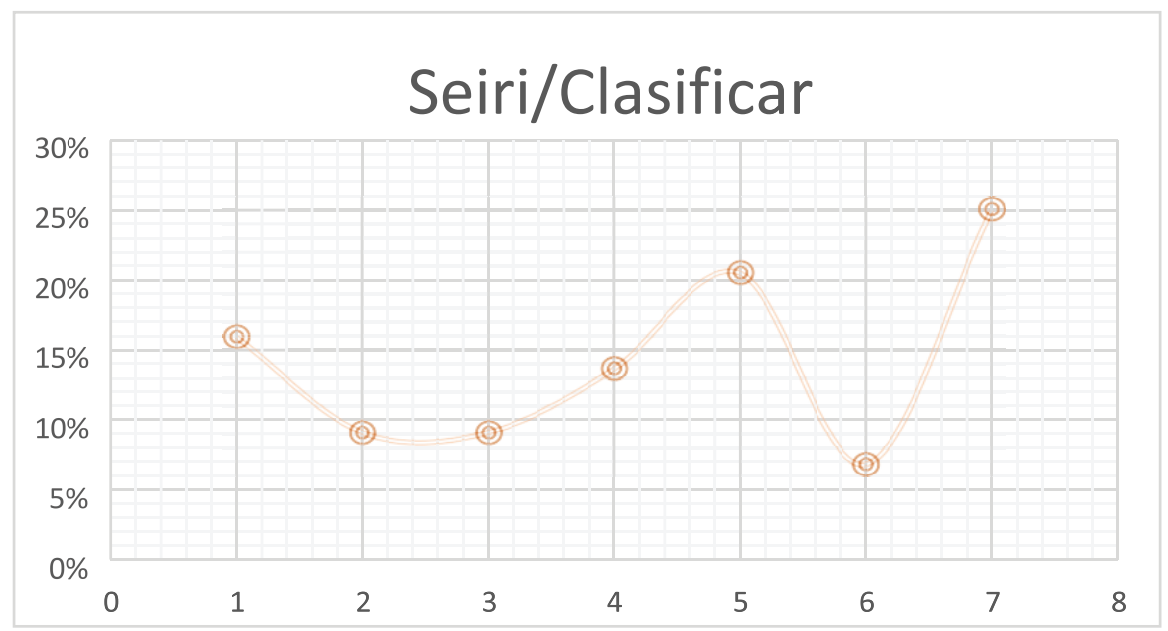

Figure 1

Datos obtenidos encuesta 5S SEIRI, Caso Comedor Politécnico. Elaborado por: Autores. Fuente: Datos obtenidos encuesta 5S SEIRI, Caso Comedor Politécnico.

SEITON: Con respecto a organización de los materiales utilizados en el comedor politécnico se obtienen resultados no tan homogéneos como se esperaba, el $5 \%$ de los encuestados otorga una calificación de 1, el 5\% una calificación de 2; el 5\% una calificación de 3; el 18\% una calificación de 4; el 
27\% una calificación de 5; el 5\% una calificación de 6 y el 36\% de los encuestados una calificación de 7, en preguntas relacionadas a ubicación de material de trabajo, seguridad, distribución de área de trabajo, orden al inicio y final de realizar el trabajo.

Table 5

Datos obtenidos encuesta 5S SEITON, Caso Comedor Politécnico.

\begin{tabular}{l|l|l|l|l|l|l|l|}
\hline Escala linker & 1 & 2 & 3 & 4 & 5 & 6 & 7 \\
\hline Frecuencias absolutas & 2 & 2 & 2 & 8 & 12 & 2 & 16 \\
\hline Frecuencas relativas & $5 \%$ & $5 \%$ & $5 \%$ & $18 \%$ & $27 \%$ & $5 \%$ & $36 \%$
\end{tabular}

Elaborado por: Autores.

Fuente: Datos obtenidos encuesta 5S SEITON, Caso Comedor Politécnico GRÁFICO 2.

\section{Seiton/Ordenar}

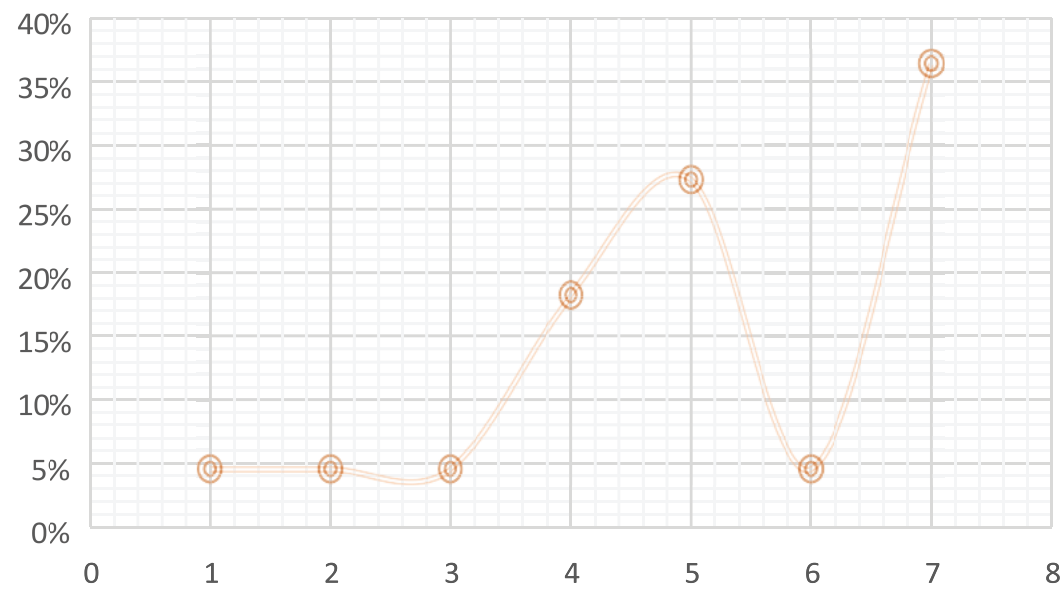

Figure 2

Datos obtenidos encuesta 5S SEITÓN, Caso Comedor Politécnico. Elaborado por: Autores. Fuente: Datos obtenidos encuesta 5S SEITON, Caso Comedor Politécnico.

SEISO: Con respecto a Limpieza en el comedor politécnico se obtienen resultados no tan homogéneos como se esperaba, el 9\% de los encuestados otorga una calificación de 1, el 7\% una calificación de 2; el 0\% una calificación de 3; el 20\% una calificación de 4; el 14\% una calificación de 5; el 18\% una calificación de 6 y el 32\% de los encuestados una calificación de 7, en preguntas relacionadas a programa de limpieza, despacho de alimentos, eliminación de fuentes de suciedad.

SEIKETSU: Con respecto a Estandarización en el comedor politécnico se obtienen resultados no tan homogéneos como se esperaba, el 5\% de los encuestados otorga una calificación de 1, el 7\% una calificación de 2; el 5\% una calificación de 3; el 25\% una calificación de 4; el 11\% una calificación de 5; el 11\% una calificación de 6 y el 36\% de los encuestados una calificación de 7, en preguntas relacionadas a ejecución de 5S, 
Table 6

Datos obtenidos encuesta 5S SEISO, Caso Comedor Politécnico.

\begin{tabular}{l|l|l|l|l|l|l|l} 
Escala linker & 1 & 2 & 3 & 4 & 5 & 6 & 7 \\
\hline Frecuencias absolutas & 4 & 3 & 0 & 9 & 6 & 8 & 14 \\
\hline Frecuencas relativas & $9 \%$ & $7 \%$ & $0 \%$ & $20 \%$ & $14 \%$ & $18 \%$ & $32 \%$
\end{tabular}

Elaborado por: Autores.

Fuente: Datos obtenidos encuesta 5S SEISO, Caso Comedor Politécnico.

\section{SEISO/LIMPIEZA}

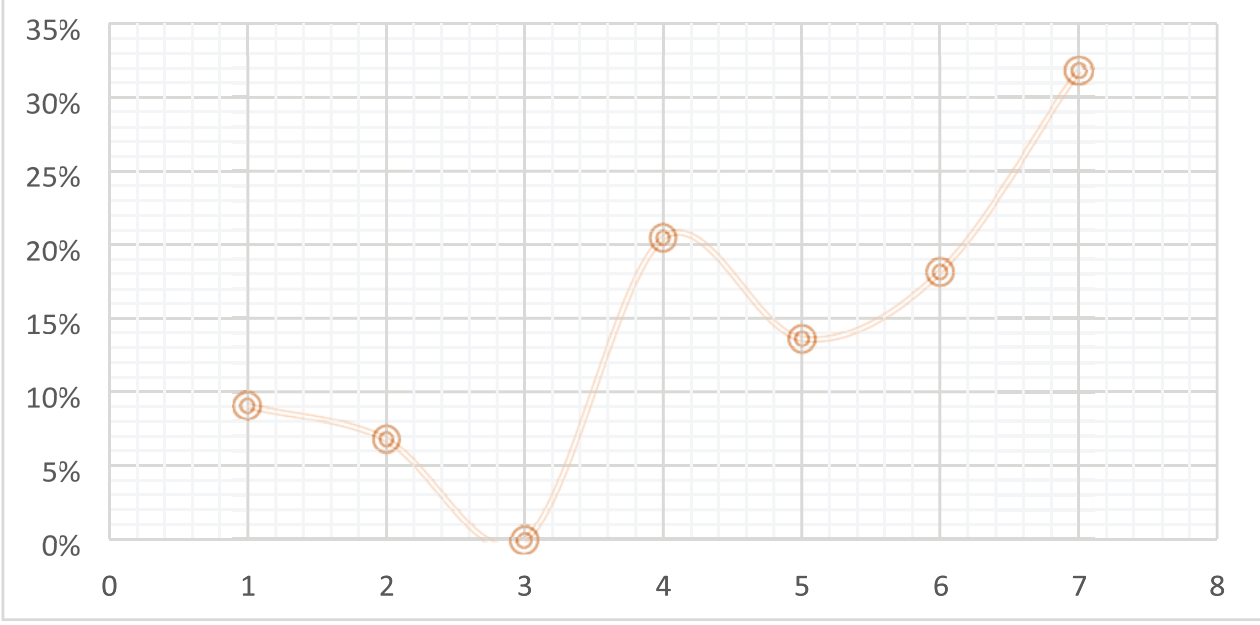

Figure 3

Datos obtenidos encuesta 5S SEISO, Caso Comedor Politécnico. Elaborado por: Autores. Fuente: Datos obtenidos encuesta 5S SEISO, Caso Comedor Politécnico.

chequeo de análisis de áreas de trabajo, estandarización de procesos, cumplimiento de normas.

\section{Table 7}

Datos obtenidos encuesta 5S SEIKETSU. Caso Comedor Politécnico.

\begin{tabular}{l|l|l|l|l|l|l|l|}
\hline Escala linker & 1 & 2 & 3 & 4 & 5 & 6 & 7 \\
\hline Frecuencias absolutas & 2 & 3 & 2 & 11 & 5 & 5 & 16 \\
\hline Frecuencas relativas & $5 \%$ & $7 \%$ & $5 \%$ & $25 \%$ & $11 \%$ & $11 \%$ & $36 \%$ \\
\hline
\end{tabular}

Elaborado por: Autores.

Fuente: Datos obtenidos encuesta 5S SEIKETSU, Caso Comedor Politécnico.

SHITSUKE: Con respecto a mantenimiento y disciplina en el comedor politécnico se obtienen resultados no tan homogéneos como se esperaba, el $5 \%$ de los encuestados otorga una calificación de 1, el $2 \%$ una calificación de 2; el 14\% una calificación de 3; el 5\% una calificación de 4; el 11\% una calificación de 5; el 7\% una calificación de 6 y el 57\% de los encuestados una calificación de 7, en preguntas relacionadas a 


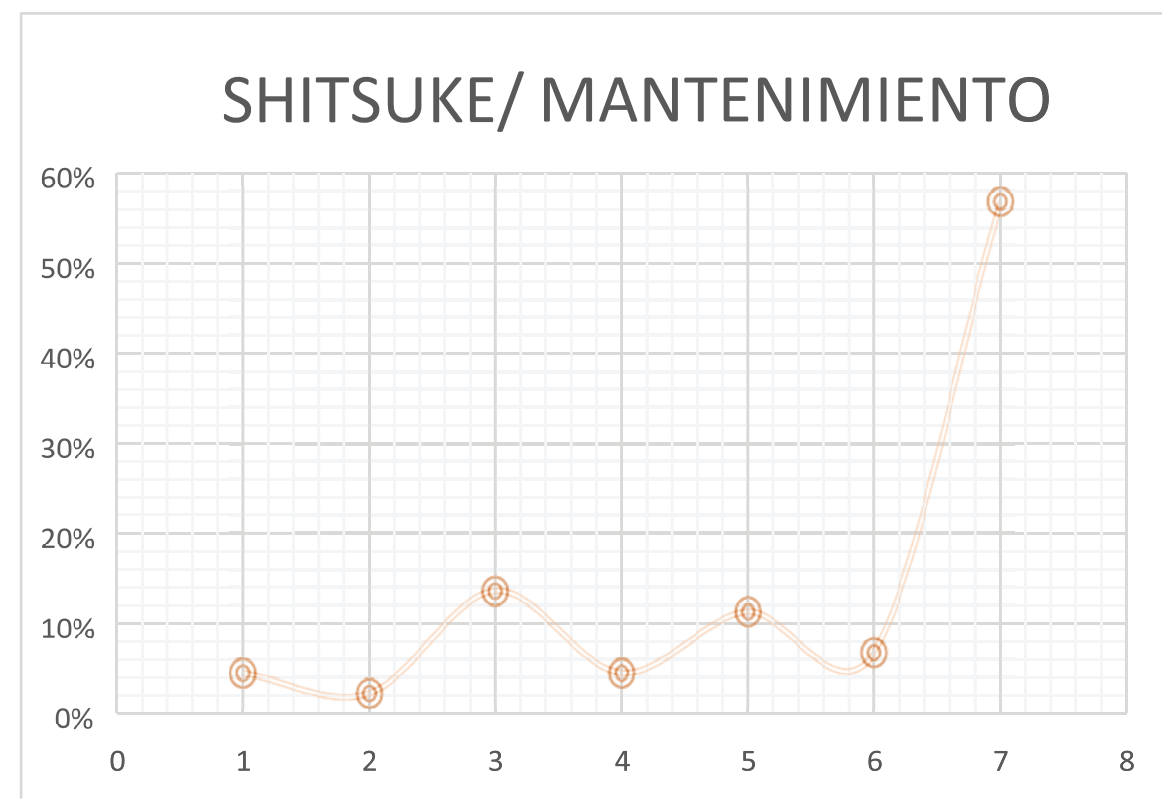

\section{Figure 4}

Datos obtenidos encuesta 5S SEIKETSU, Caso Comedor Politécnico. Elaborado por: Autores. Fuente: Datos obtenidos encuesta 5S SEIKETSU, Caso Comedor Politécnico.

utilización de uniforme, equipo de protección, cumplimiento de horarios de trabajo, cultura organizacional.

\section{Table 8}

Datos obtenidos encuesta 5S SHITSUKE. Caso Comedor Politécnico.

\begin{tabular}{l|l|l|l|l|l|l|l} 
Escala linker & 1 & 2 & 3 & 4 & 5 & 6 & 7 \\
\hline Frecuencias absolutas & 2 & 1 & 6 & 2 & 5 & 3 & 25 \\
\hline Frecuencas relativas & $5 \%$ & $2 \%$ & $14 \%$ & $5 \%$ & $11 \%$ & $7 \%$ & $57 \%$
\end{tabular}

Elaborado por: Autores.

Fuente: Datos obtenidos encuesta 5S SHITSUKE, Caso Comedor Politécnico.

\section{Discusión}

A continuación, se presentan los problemas más relevantes encontrados: Seiri (Clasificación)

1. Existen problemas innecesarios en el puesto de trabajo (mobiliario, equipos de comedor);

2. Insufientes materiales (lencería, cubiertería, cristalería);

3. Aplicación de procedimeintos para eliminar material innecesario.

Seiton (Orden) 


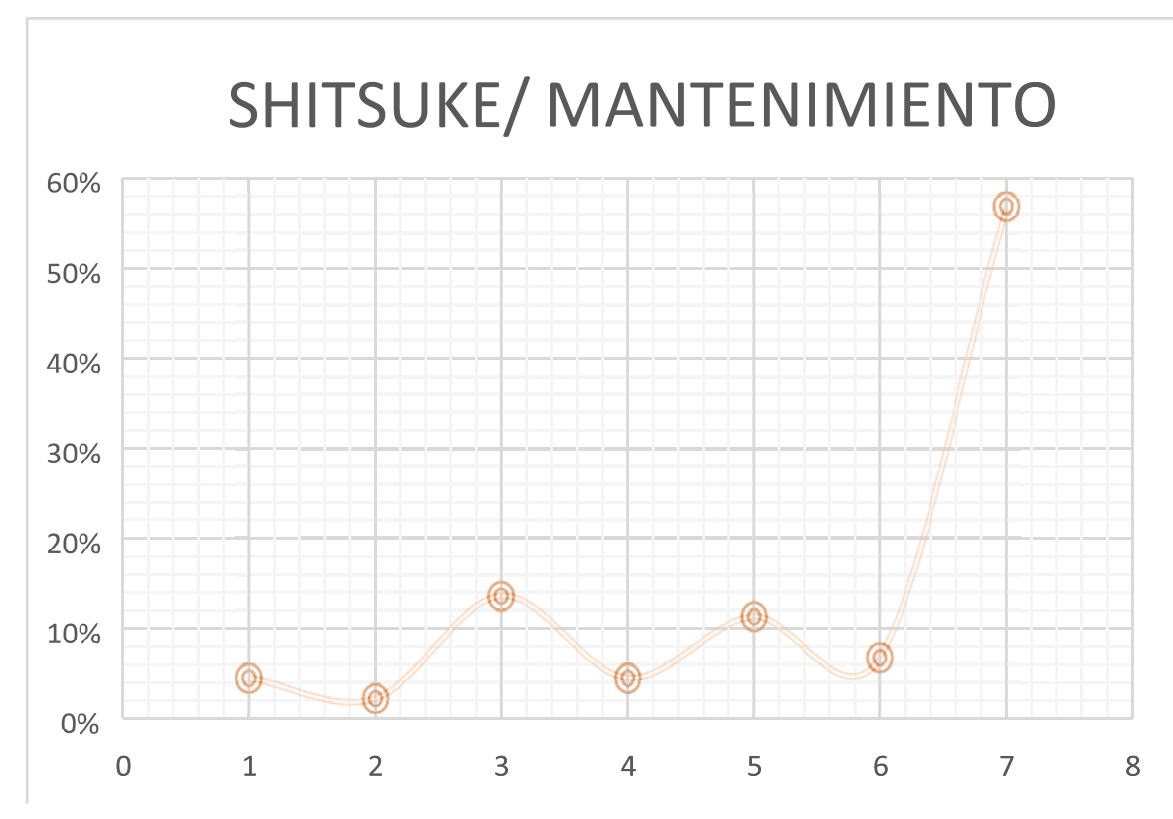

\section{Figure 5}

Datos obtenidos encuesta 5S SEIKETSU, Caso Comedor Politécnico. Elaborado por: Autores. Fuente: Datos obtenidos encuesta 5S SHITSUKE, Caso Comedor Politécnico.

1. Los materiales no tienen una ubicación específica en el puesto de trabajo;

2. El personal mantiene el orden durante la realización y culminación de su trabajo.

Seiso (Limpieza)

1. Programa de limpieza en el área de trabajo;

2. El programa de limpieza abarca todas las áreas de trabajo;

3. Programas para eliminar fuentes de suciedad.

Seiketsu (Estandarización)

1. Utilización de chequeo para analizar el área de trabajo;

2. Las normas, reglamentos y disposiciones se aplican a todos de la misma manera.

Shitsuke (Mantenimiento)

1. Horarios establecidos en su área de trabajo;

2. Cultura Organizacional. 


\section{Conclusiones}

Obteniendo la calificación más alta de 7, en lo referente Seiri (clasificación) 25\%, Seiton (Orden) 36\%, Seiso (Limpieza) 32\%, Seiketsu (Estandarización) 36\%, Shitsuke (Mantenimiento) 57\%, de esta manera de concluye que en Shitsuke donde existe mayor compromiso de los empleados, y en Seiri es la que menor calificación se obtiene y con la calificación más baja 1, en lo referente a Seiri (clasificación) 16\%, Seiton (Orden) 5\%, Seiso (Limpieza) 9\%, Seiketsu (Estandarización) 5\%, Shitsuke (Mantenimiento) 5\%.

\section{References}

[1] Rovira C. Implementación de sistemas de gestión. 2011. Obtenido de: http://www. op-group.net/

[2] Barcia F, Hidalgo D. Implementación de una metodología con la técnica 5S para mejorar el área de metricería de una empresa extrusora de aluminio. Revista Tecnológica ESPOL. 2006;18(1):69-75.

[3] Juarez H. Propuesta para implementar metodología 5S en el departamento de cobros de la de subdelegación Veracruz. 2009. Obtenido de: http://www.redalyc. org/pdf/679/67932397009.pdf

[4] Lefcovich M. Kaizen y su aplicación en instituciones de salud: Su aplicación en materia de mejoramiento continuo en los niveles de calidad, productividad y costos. 2009. Obtenido de: Retrieved from https://ebookcentral.proquest.com

[5] Lefcovich M. L. Las 5 S Plus. 2009. ProQuest Ebook Central. 2009. Obtenido de https://ebookcentral.proquest.com/lib/espochsp/detail.action?docID=3181073.

[6] Rodriguez del Pozo F. Mejoramiento contínuo de la calidad. 1992. Obtenido de https://dialnet.unirioja.es/buscar/documentos?querysDismax.DOCUMENTAL_ TODO=MEJORAMIENTO\{\%\}20CONTINUO\&filtros.DOCUMENTAL_FACET_ ENTIDAD=artrev

[7] Álvarez D. Procedimiento para el mejoramiento de los procesos en el hotel sunbeach. 2011. Obtenido de Retrieved from https://ebookcentral.proquest.com

[8] Sumanth D. Administración para la productividad total. Mexico: Continental. México; 1999.

[9] Kurosawa K. Medición y análisis de la productividad a nivel de empresa. Venezuela: YRGOY; 1983.

[10] Prokopenko J. 1987.

[11] Mercado E. Productividad, base de la Competitividad. México: LIMUSA; 1998.

[12] Gutiérrez H. Calidad total y productividad. México.: Mc Graw Hill; 2006.

[13] Lucey J. Productivity: What's going on in europe part II. Management Services. 2007. 
[14] Velasquez Y, Rodriguez C, Guaita W. Modelo de los factores de productividad que afectan la productividad. 2012. Obtenido de https://dialnet.unirioja.es/ buscar/documentos?querysDismax.DOCUMENTAL_TODO=productividad\&filtros. DOCUMENTAL_FACET_ENTIDAD=artlib

[15] Sarmiento S. Competitividad regional. 2008. Obtenido de: https://dialnet.unirioja.es/ buscar/documentos?querysDismax.DOCUMENTAL_TODO=competitividad 